\title{
STABILITY OF SYMPTOM CLUSTERS AND SENTINEL SYMPTOMS DURING THE INITIAL PHASE OF ADJUVANT CHEMOTHERAPY
}

\author{
Jiyeon Lee ${ }^{1}$, Mira Park ${ }^{2}$, Sun Young Rha ${ }^{3}$
}

${ }^{1}$ Chungnam National University, College of Nursing, Daejeon, Republic of Korea

2 Eulji University, College of Medicine, Daejeon, Republic of Korea

${ }^{3}$ Yonsei University, College of Medicine, Seoul, Republic of Korea

\section{Introduction}

Top priorities in symptom cluster studies include confirming stable groups of symptoms and identifying sentinel symptoms. Understanding the stability of symptom clusters and identifying sentinel symptoms would facilitate effective symptom management.

\section{Objectives}

This study aimed to evaluate the stability of symptom clusters and to identify sentinel symptoms during the initial phase of adjuvant chemotherapy (CTx).

\section{Methods}

Design: Secondary data analysis of the Paradigm Shift in ChemotherapyInduced Nausea and Vomiting (PS-CINV) study.

Sample: A total of 209 cancer patients receiving adjuvant CTx who completed symptom evaluation for the $1^{\text {st }}$ and $2^{\text {nd }}$ cycles.

Measurement:The severity of 20 symptoms on a 0 to 10 numeric rating scale. Analysis: Principal component analysis and hierarchical cluster analyses to identify symptom clusters using SPSS 24.0 and SAS 9.4 .Principal variable analysis to identify sentinel symptoms using $R$ program 3.4.I. Principal variable analysis considered minimum trace of partial variances of the unselected variables unexplained by the selected variables was utilized.

\section{Results}

Four symptom clusters were identified in the $\mathrm{I}^{\text {st }}$ cycle: a physicalpsychological (pain, dyspnea, sleep disturbance, anxiety, depression), a gastrointestinal (nausea, loss of appetite, taste change), a fatigue-cognitive (fatigue, difficulty concentrating, drowsiness), and a urosexual (urinary problem, sexual problem) symptom cluster.

In the $2^{\text {nd }}$ cycle, stable symptom clusters were identified, whereas the physical-psychological and fatigue-cognitive symptom cluster were merged together resulting in 3 clusters.

The identified sentinel symptoms were anxiety, loss of appetite and fatigue in the $1^{\text {st }}$ cycle, and loss of appetite, depression, and fatigue in the $2^{\text {nd }}$ cycle.

\section{Conclusions}

Stable symptom clusters were identified during the initial phase of adjuvant CTx. The identified sentinel symptoms will facilitate efficient symptom management. Symptom management needs to be focused on managing emotional symptoms, appetite and fatigue.

\section{Acknowledgement}

The current study was supported in part by the Basic Science Research Program through the National Research Foundation of Korea, funded by the Ministry of Education, Science, and Technology (20I2RIAIAI0I0I07 and 20I5RIAIAIA0500 I342), in part by the Chungnam National University Research Fund (2016-1793-0I), and in part by the National R\&D Program for Cancer Control, Ministry of Health and Welfare, Republic of Korea (1520190).

\begin{tabular}{lcccccc}
\hline & \multicolumn{3}{c}{ Ist cycle } & \multicolumn{3}{c}{ 2nd cycle } \\
\cline { 2 - 7 } & $\begin{array}{c}\text { Prevalence } \\
(\%)\end{array}$ & Mean & SD & $\begin{array}{c}\text { Prevalence } \\
(\%)\end{array}$ & Mean & SD \\
\hline difficulty concentrating & $162(77.5)$ & 3.36 & 2.81 & $168(80.4)$ & 3.40 & 2.66 \\
drowsiness & $174(83.3)$ & 3.93 & 2.92 & $180(86.1)$ & 3.99 & 2.76 \\
fatigue & $190(90.9)$ & 4.87 & 2.87 & $199(95.2)$ & 4.97 & 2.74 \\
sleep disturbance & $153(73.2)$ & 3.76 & 3.35 & $165(78.9)$ & 3.67 & 2.9 \\
anxiety & $134(64.1)$ & 2.58 & 2.81 & $136(65.1)$ & 2.48 & 2.64 \\
depression & $126(60.3)$ & 2.33 & 2.66 & $134(64.1)$ & 2.46 & 2.58 \\
pain & $134(64.1)$ & 2.70 & 3.01 & $136(65.1)$ & 2.58 & 2.71 \\
dyspnea & $115(55.0)$ & 2.02 & 2.63 & $125(59.8)$ & 2.30 & 2.73 \\
mucositis & $78(37.3)$ & 1.22 & 2.26 & $106(50.7)$ & 1.84 & 2.53 \\
taste change & $166(79.4)$ & 4.31 & 3.48 & $180(86.1)$ & 4.53 & 3.33 \\
loss of appetite & $178(85.2)$ & 5.00 & 3.51 & $180(86.1)$ & 4.78 & 3.28 \\
nausea & $176(84.2)$ & 4.41 & 3.34 & $180(86.1)$ & 4.39 & 3.18 \\
vomiting & $78(37.3)$ & 1.39 & 2.51 & $83(39.7)$ & 1.46 & 2.46 \\
constipation & $125(59.8)$ & 3.33 & 3.60 & $134(64.1)$ & 2.66 & 2.94 \\
diarrhea & $67(32.1)$ & 1.37 & 2.58 & $82(39.2)$ & 1.37 & 2.38 \\
urinary problem & $66(31.6)$ & 1.30 & 2.54 & $74(35.4)$ & 1.24 & 2.29 \\
sexual problem & $50(23.9)$ & 1.07 & 2.49 & $70(33.5)$ & 1.51 & 2.75 \\
skin and nail change & $52(24.9)$ & 0.77 & 1.87 & $105(50.2)$ & 2.02 & 2.77 \\
alopecia & $66(31.6)$ & 0.91 & 2.08 & $161(77.0)$ & 4.87 & 4.03 \\
neuropathy & $111(53.1)$ & 2.13 & 2.77 & $148(70.8)$ & 3.14 & 3.03 \\
\hline
\end{tabular}

Table I. Symptom prevalence and severity in the $\mathrm{I}^{\text {st }}$ and $2^{\text {nd }} \mathrm{CTx}$ cycles

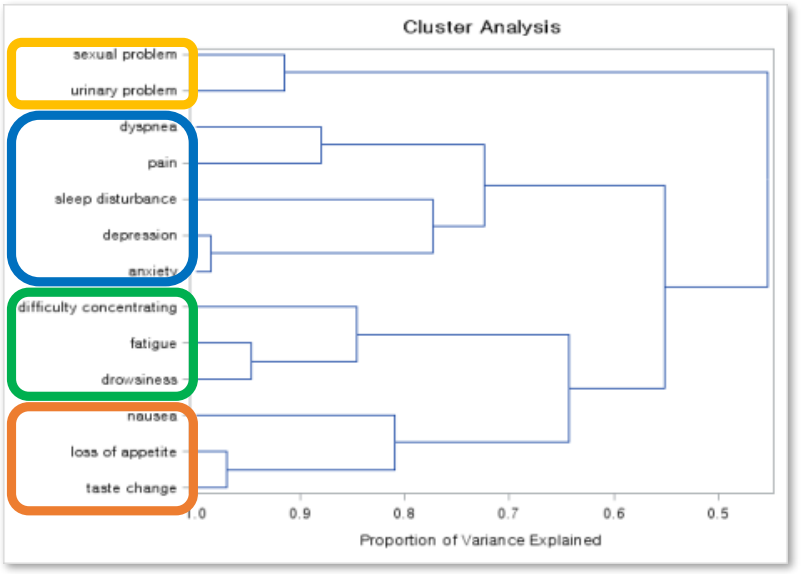

Figure I. Symptom clusters in the $\left.\right|^{\text {st }}$ cycle

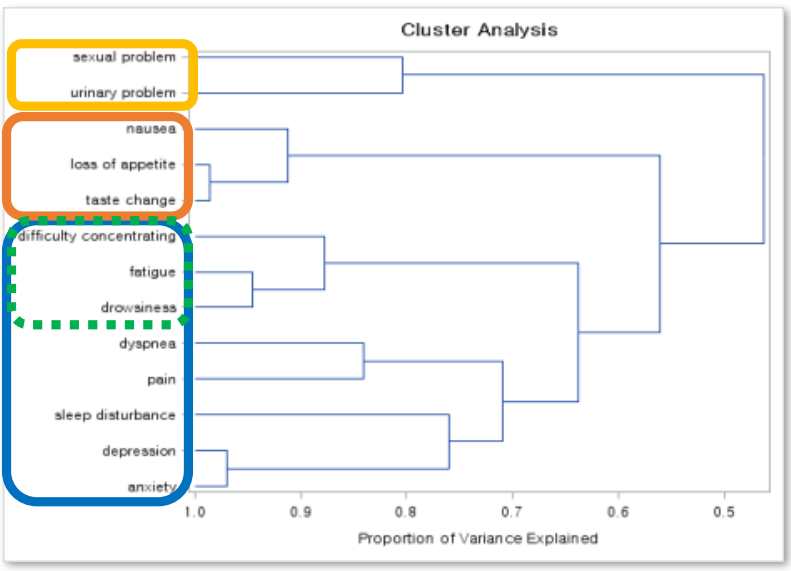

Figure 2. Symptom clusters in the $2^{\text {nd }}$ cycle 